\title{
Emphysematous pyelitis, ureteritis and cystitis
}

\author{
James W. Wong, MD; Kenneth T. Pace, MD, FRCSC ${ }^{\dagger}$
}

Can Urol Assoc J 2010;4(6):E148-E149

A 36-year-old man with a history of gout and a congenital solitary right kidney with chronic hydronephrosis and non-obstructive megaureter of unknown etiology presented initially with a stone on KUB and flank pain. A computed tomography $(\mathrm{CT})$ scan showed a right-sided calculus which was treated with extracorporeal shock wave lithotripsy and alkalinization therapy. Several weeks posttreatment, he presented to the emergency department with cloudy urine and dysuria. His urinalysis demonstrated pyruia and hematuria. He was empirically started on ciprofloxacin, but returned to the emergency department several days later with fevers, chills and rigors but no flank pain. His creatinine had risen from $109 \mathrm{mmol} / \mathrm{L}$ at baseline to $266 \mathrm{mmol} / \mathrm{L}$, and his white blood cell count was $15.6 \times 10^{6} / \mathrm{L}$. A CT scan failed to show any residual calculi; however, it demonstrated emphysematous pyelitis (Fig. 1, arrow) with concomitant emphysematous ureteritis and cystitis (Fig. 2, arrows). His

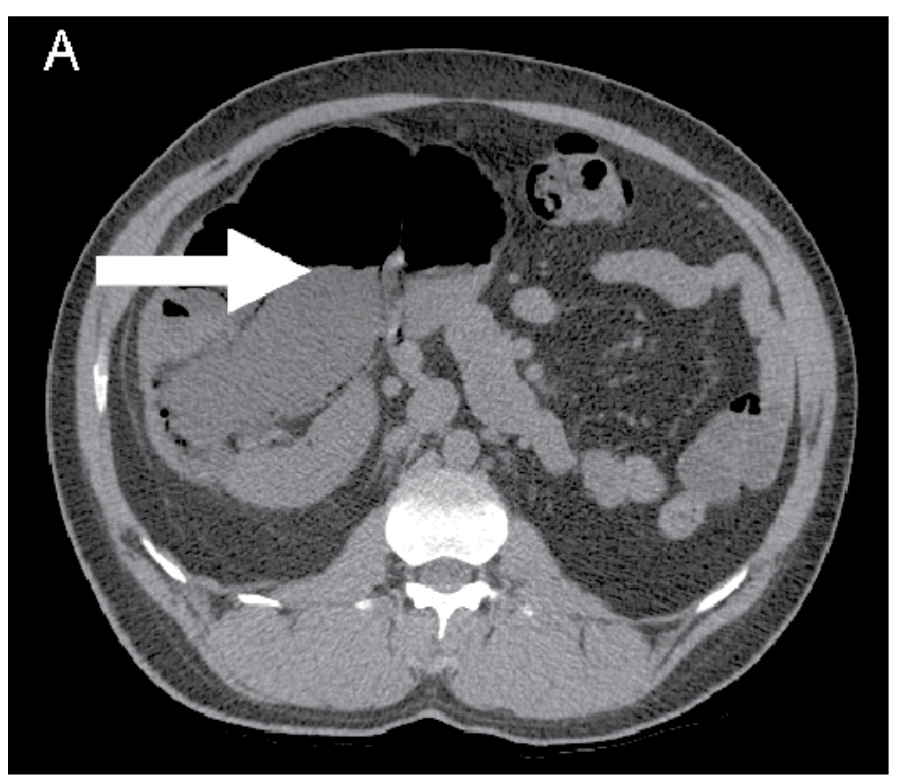

Fig. 1. The computed tomography scan with the arrow pointing to the emphysematous pyelitis. urine culture revealed a multi-drug resistant $E$ Coli and a percutaneous nephrostomy tube was arranged after a ureteric stent insertion was unsuccessful. His pre-nephrostogram scout x-ray showed a collecting system full of gas (Fig. 3, arrows demonstrating gas in ureter and renal pelvis). He was treated with nephrostomy tube drainage and intravenous ceftriaxone. Within 5 days, he had complete clinical and radiographical resolution of the gas in his collecting system, and his renal function returned to baseline. Subsequent pressure-flow studies of urinary drainage via the nephrostomy tube (Whittaker test) showed no evidence of ureteric obstruction. It is important to distinguish emphysematous pyelitis (gas in the collecting system) from emphysematous pyelonephritis (gas in the renal parenchyma), as the latter can be more severe.

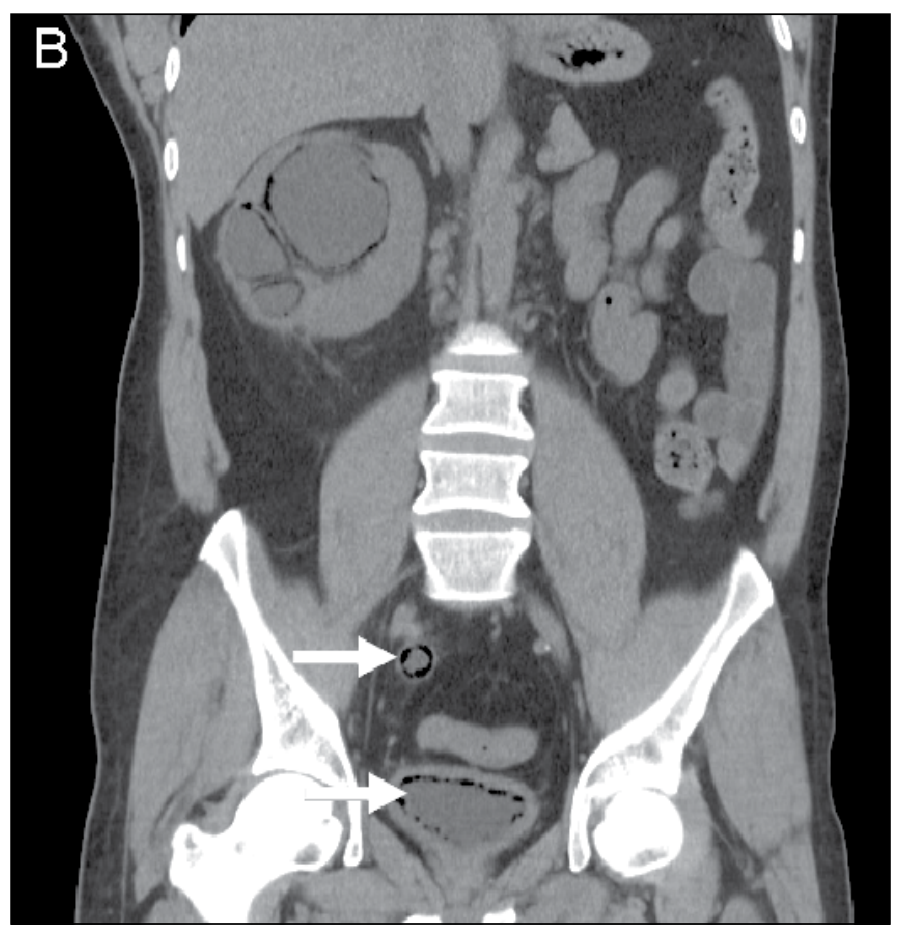

Fig. 2. The computed tomography scan with the arrows pointing to the emphysematous ureteritis and cystitis. 


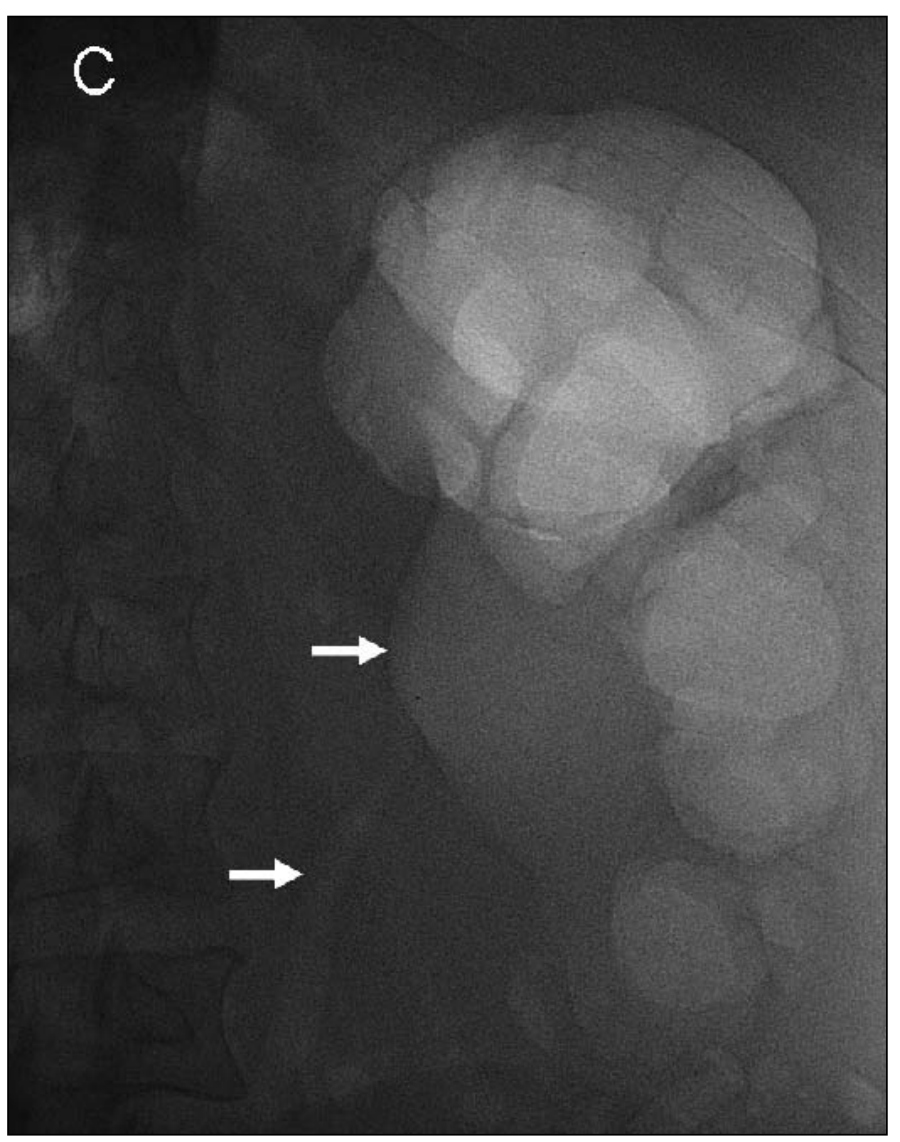

Fig. 3. The patient's pre-nephrostogram with the arrows demonstrating gas in the ureter and renal pelvis.
Empysematous pyelitis is due to $E$. coli infection in $69 \%$ of cases and typically responds promptly to medical therapy. ${ }^{1}$

¿Department of Urology and the Centre for Applied Urological Research, Queen's University, Kingston, ON; 'Department of Urology, St. Michael's Hospital, Toronto, ON

Competing interests: None declared for Dr. Wong. Dr. Pace is a consultant for Baxter Pharmaceutical. He has a grant pending from Sanofi-Synthelabo for another RCT for which he is the investigator. He has given talks for both Baxter Pharmaceutical and for Pfizer Canada. He is the director for an Endourology Society fellowship that receives fellowship funding from Cook Canada.

This paper has been peer-reviewed.

\section{Reference}

1. Huang JJ, Tseng CC. Emphysematous pyelonephritis: clinicoradiological classification, management, prognosis, and pathogenesis. Arch Intern Med 2000;160:797-805.

Correspondence: Dr. James W. Wong, 1106-763 Bay St., Toronto, ON M5G 2R3; jamesmd@ gmail.com 